\title{
Cellular binding partners of the human papillomavirus E6 protein
}

\author{
Sandy S. Tungteakkhun • \\ Penelope J. Duerksen-Hughes
}

Received: 12 September 2007 / Accepted: 13 December 2007/Published online: 3 January 2008

(C) The Author(s) 2007

\begin{abstract}
The high-risk strains of human papillomavirus (HR-HPV) are known to be causative agents of cervical cancer and have recently also been implicated in cancers of the oropharynx. E6 is a potent oncogene of HR-HPVs, and its role in the progression to malignancy has been and continues to be explored. E6 is known to interact with and subsequently inactivate numerous cellular proteins pivotal in the mediation of apoptosis, transcription of tumor suppressor genes, maintenance of epithelial organization, and control of cell proliferation. Binding of E6 to these proteins cumulatively contributes to the oncogenic potential of HPV. This paper provides an overview of these cellular protein partners of HR-E6, the motifs known to mediate oncoprotein binding, and the agents that have the potential to interfere with E6 expression and activity and thus prevent the subsequent progression to oncogenesis.
\end{abstract}

\section{Introduction}

Human papillomavirus (HPVs) are small, double-stranded DNA viruses that preferentially infect epithelial tissues, including those of the anogenital tract. It is also now reported that a subset of these HPVs infect the oropharynx [73]. Of the more than 100 different types of HPV that have been identified, about 40 are involved in genital tract infection. These HPVs can be classified as either high-risk (HR) or low-risk (LR) depending upon the transforming

S. S. Tungteakkhun · P. J. Duerksen-Hughes ( $\bowtie)$

Department of Basic Sciences,

Loma Linda University School of Medicine,

Loma Linda, CA 92354, USA

e-mail: pdhughes@1lu.edu potential of the virus. Infection with LR-HPVs (HPV 6 or 11), for instance, can result in the proliferation of epithelial cells, which manifests itself as warts or papillomas on the skin. These symptoms, however, are generally self-limiting and do not lead to malignancy. Once the virus has entered its host through a disturbance in the epithelial barrier, the principal targets of the virus are keratinocytes in the basal layer of squamous epithelia [1, 50, 65, 74]. In infected cells, the viral genome is either maintained as an episome or, in rare instances, becomes integrated into the host genome, where its life cycle becomes closely linked to the differentiation course of the host cell. In general, benign tissues display episomal viral DNA, while it is after DNA integration has taken place that HPV has the greatest potential to induce oncogenicity. Although viral DNA integration is a rare event, its occurrence may initiate a series of events resulting in the genomic instability that can facilitate subsequent cellular immortalization and transformation. In this way, infection with HR-HPVs (HPV 16 or 18) may lead to cervical intraepithelial neoplasia (CIN) in some women. In fact, the HR strains 16 and 18 are present in $>90 \%$ of cervical cancer cases and have also been implicated in head and neck squamous cell carcinomas, with HPV 16 being the most common viral type [28, 41, 99].

Cancer is a major health concern in many developed countries. In 2004, it was the second leading cause of death in the US, following cardiovascular disease [55]. Currently, HPV-induced cervical cancer is the second most common cancer and the fifth leading cause of cancer-related deaths among women worldwide [14, 73, 90]. Presently, there are close to half a million cases of cervical cancer worldwide. Due to these staggering statistics and in order to enable the prevention of future HPV-induced cancers, companies such as Merck and GlaxoSmithKline have developed HPV vaccines that have the potential to eliminate up to $70 \%$ of 
invasive cervical cancers and up to $90 \%$ of genital warts. These prophylactic vaccines, however, are aimed at preparing the immune system for possible future encounters with the virus and cannot be used for the treatment of already established virus infection or the cancers they cause.

HPVs have a genome that is divided into three regions: an early region (E), a late region (L), and a non-coding long control region (LCR). The $\mathrm{E}$ region encodes six nonstructural proteins: E1, E2, E4, E5, E6, and E7. The L region encodes two structural proteins: $\mathrm{L} 1$ and $\mathrm{L} 2$. The $\mathrm{E} 1$, E2, E4, and E5 proteins are required for viral DNA replication, the E6 and E7 proteins cooperate to transform and immortalize cells, and the L1 and L2 proteins are needed for the production of viral particles [68, 82]. As previously mentioned, integration of HR-HPVs into the host cell genome can result in genomic instability and immortalization of the host cell. Viral DNA integration is often accompanied by disruption of the E2 gene, and, less frequently, of E1. The E2 protein is known to function, among other things, as a transcriptional repressor of both E6 and E7 expression. It has also been observed that the E1 gene is frequently interrupted in carcinomas, suggesting that the E1 gene product may also be a negative regulator of viral transcription [95]. Because the E2 protein is a repressor of E6 and E7 expression, the loss of E2 leads to an uncontrolled increase in the levels of these oncoproteins. The low levels of E6 and E7 that are seen early in the normal viral life cycle enable the initially low number of virus-infected cells to survive and expand [30]. Once integration occurs and levels of E2 drop, elevated levels of E6 and E7 can result, which in turn enable cellular transformation. It should be noted that many or most of these integration events are likely to be non-productive, due to the loss of the early region splice acceptor and polyadenylation sites [109]. Most studies that employ over-expression of these oncoproteins thus create conditions that are most analogous to the latter set of conditions.

E6 and E7 together are effective in promoting the immortalization of human foreskin keratinocytes in culture, suggesting that they significantly contribute to the oncogenic potential of HR-HPVs [80]. Results from numerous studies demonstrate that interactions of E6 and E7 with the tumor suppressor p53 and several members of the retinoblastoma family proteins including pRb, p107, and p130, respectively, lead to the inhibition of apoptosis and a subsequent increase in the replication of transformed cells [8, 33, 79]. In addition, interactions between HR-HPV oncoproteins and endogenous cellular proteins have been shown to trigger cell cycle deregulation, a critical component in the progression to cancer [40]. Other research results have reported on the significance of the viral oncoprotein E6 from HR-HPVs in mediating cellular transformation, supporting tumorigenesis, interfering with epithelial organization, and activating telomerase. A thorough understanding of the interactions of E6 with its numerous cellular protein partners, as well as the consequences of these interactions, allows for the possibility of developing therapeutic approaches to impede the activity of the oncoprotein.

\section{The E6 oncoprotein}

The HPV 16 E6 protein consists of 151 amino acids, with two zinc fingers characterized by the CXXC motif. Although it is a relatively short protein, E6 has proven difficult to study due to the inability of the protein to be isolated in a native, soluble form. E6 consists of a high content of $\alpha$-helical and $\beta$-sheet secondary structures. These properties give rise to a protein that is both unstable and insoluble once purified [86]. Recently, however, a model of the structure of HPV 16 E6 has been proposed using an E6 mutant in which six non-conserved cysteines were replaced with serine [87]. These substitutions not only allowed for the purification of a soluble protein, but also produced a protein that retained several tested biological activities of its wild-type predecessor, including its ability to degrade p53 and to associate with its known protein partner E6-AP. The availability of the three-dimensional structure of E6 allows for the visualization of the potential residues that mediate binding to its cellular protein partners. It is important to note that the proposed model is based on the sequence homology between the $\mathrm{N}$ and $\mathrm{C}$-terminal halves of the protein and the solved structure of the C-terminal domain of HPV 16 E6. Some significant aspects of the native structure, such as the interactions between the two domains and the structure of the two domain boundaries, as well as the binding pocket for leucine-rich peptides, therefore, remain incompletely defined. However, although the derived structure may not accurately model the E6 protein in all respects, it does provide a framework for the potential development of agents to specifically block interactions between E6 and its protein partners. The validity of these agents would then need to be further tested for biological significance in the presence of the viral genome.

E6 is one of the earliest genes expressed following viral infection and is one of two principal oncogenes of HPV, along with E7. E6 was first classified as an oncogene when in vitro and in vivo studies demonstrated that E6 expression can lead to hyperproliferation of cells, loss of epithelial cell differentiation, and tumor formation. Additionally, studies with cervical tumors revealed that not only is E6 expressed immediately following the initial transformation, but expression is retained and can be detected long after the transforming event [17, 77]. Expression of the HR-E6 protein alone is sufficient to immortalize 
primary human mammary epithelial cells [6, 118], and E6 in cooperation with E7 can induce immortalization of primary human foreskin keratinocytes in culture [48, 117]. Studies with LR-E6 reveal that these proteins cannot induce the immortalization of these cells and are therefore, inactive [48]. Progression to malignancy following viral infection is thus reserved for HR-HPV strains.

To further substantiate these findings and to underline the importance of E6 in HPV-induced carcinogenesis, transgenic mice expressing the E6 protein have been generated. It has been found that formation of tumors is highly dependent upon where the oncogene is expressed, as expression of E6 in basal layers of squamous epithelium leads to the development of squamous epithelial neoplasia [4]. In addition, results from experiments with transgenic mice expressing a mutant form of E6, unable to interact with some of its cellular protein partners, revealed a significant reduction in the ability of E6 to induce cervical carcinogenesis [100]. Taken together, these data demonstrate the potency of the oncoprotein E6 in contributing to the malignant phenotype that may arise following successful infection with HR-HPVs, such as HPV 16.

\section{Protein partners of HPV 16 E6}

The current literature provides us with abundant information regarding cellular proteins to which E6 binds and inactivates in order to support cell transformation. Most of the cellular binding partners of E6 have been identified by over-expressing these proteins in vivo. Therefore, although binding has been determined for each of the protein partners, the functional significance of many of these interactions at normal expression levels requires further evaluation. Nevertheless, results garnered from these overexpression studies reveal that E6 interacts with a number of different proteins that mediate the apoptotic pathway, regulate transcription, and mediate chromosomal stability, epithelial organization, differentiation, cell-cell adhesion, polarity, and proliferation control in the infected cell (Fig. 1). Interactions of E6 with these various proteins contribute to the overall effectiveness of E6 in the promotion of HPV oncogenicity.

\section{Mediators of apoptosis}

The first activity identified for HR-HPV E6 proteins was its ability to form a complex with the tumor suppressor p53 in vivo and to transform cells in vitro [119]. To avoid apoptotic clearance, the E6 protein of oncogenic HPVs binds to and inactivates p53, leading to its degradation and ablating its ability to negatively regulate cell cycle progression. Protein-protein interaction studies with E6 and p53 demonstrate that E6 binding impedes the normal physiological function of p53. Moreover, the E6-mediated inhibition of
Fig. 1 Representation of cellular proteins affected by HR-E6. "Asterisk" Designates proteins where the influence of HR-E6 is at the level of transcription; proteins without an asterisk are those to which HR-E6 has been shown to bind

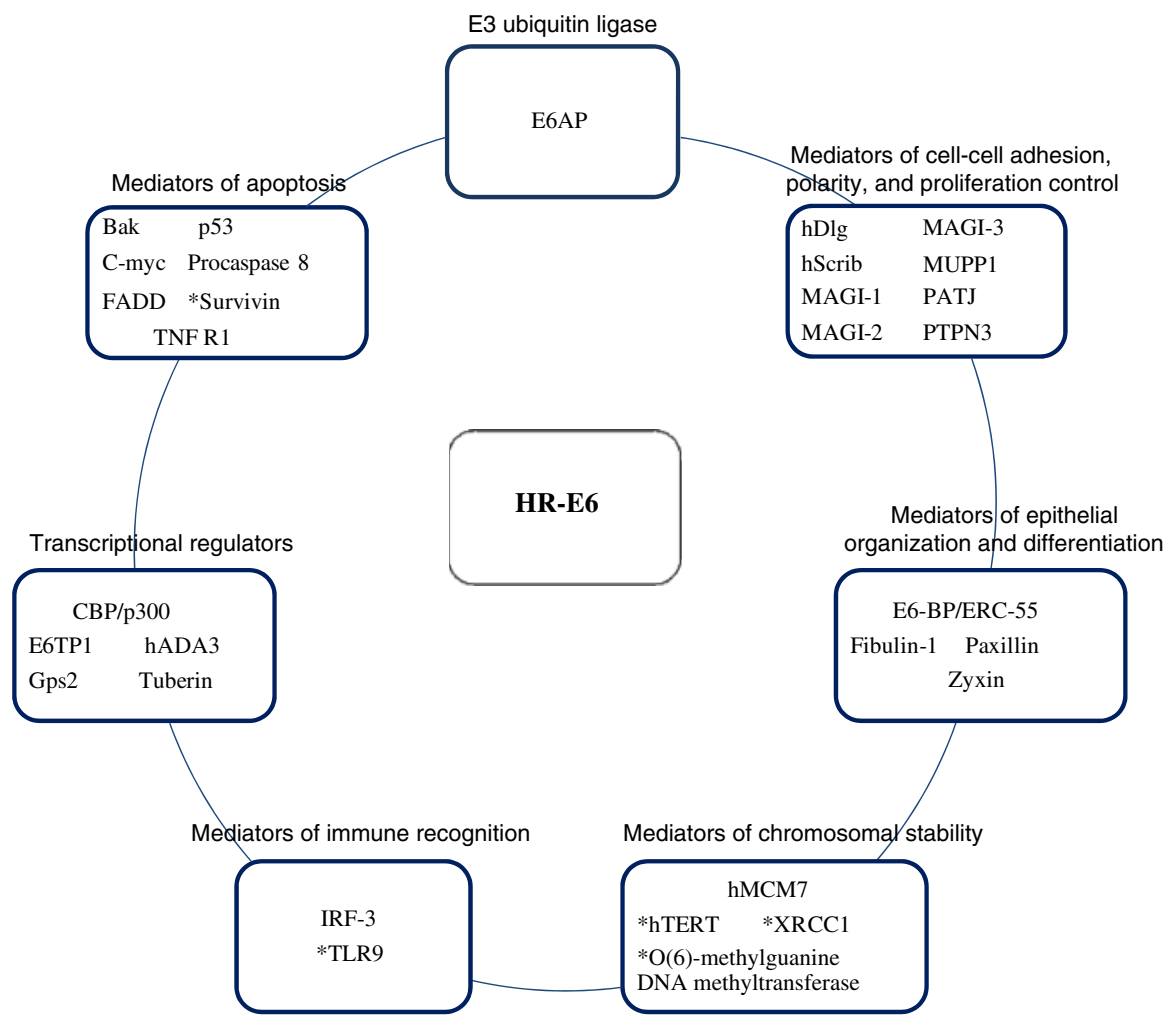


p53 activity facilitates the survival of transformed cells $[23,67]$. To determine whether the ability of E6 to mediate p53 degradation is critical for the immortalization of mammary epithelial cells (MECs), mutations that render E6 unable to mediate p53 degradation were introduced into the oncogene and tested. Dalal et al. report that p53 turnover is required for HPV-E6-induced immortalization of MECs [24]. E6-mediated degradation of p53 requires the formation of a trimolecular complex consisting of E6, the 100-kDa cellular E6-associated protein E6-AP, and p53. The interaction between E6 and E6-AP gives rise to the ubiquitin ligase moiety that directs p53 degradation [98, 108].

In 1995, Pan and Griep reported that in p53-null mice expressing the HPV 16 E6 protein, apoptosis was inhibited, suggesting that E6 can also inhibit apoptosis by means independent of p53 [89]. Indeed, E6 is now known to bind to the pro-apoptotic protein Bak, mediate its degradation via the proteasome, and reduce its ability to trigger apoptosis [111]. Similarly, E6 prompts the degradation of the pro-apoptotic c-myc protein, resulting in sustained proliferation of infected cells [57]. E6 can also exert its effects on other apoptosis-related proteins at the transcriptional level, as in the case of the anti-apoptotic protein survivin. E6 is reported to significantly up-regulate survivin promoter activity, giving rise to the suppression of apoptosis [12]. Additional components of the host apoptotic machinery to which E6 has been reported to bind include the tumor necrosis factor receptor 1 (TNF R1) [34], the adaptor molecule Fas-associated death domain (FADD) [35], and procaspase 8 [36]. E6 binds to the death effector domains (DEDs) of FADD and procaspase 8 and, in doing so, mediates the accelerated degradation of both. Thus, binding of E6 to these proteins make them unable to efficiently participate in transmission of the apoptotic signal, giving rise to the protection observed from apoptosisinducing stimuli.

\section{Transcriptional regulation}

In addition to the proteins that mediate apoptosis, E6 also interacts with proteins involved in transcription control. Yeast two-hybrid analyses, for instance, demonstrate that E6 binds to E6TP1. E6TP1 exhibits high homology to GTPase-activating proteins (GAPs) which are known to function as tumor suppressors [37, 38]. In this way, inactivation of E6TP1 by E6 may contribute to viral oncogenesis. Results from similar experiments with hADA3, the human homolog of yeast transcriptional coactivator yADA3, show that E6 also binds to this protein [52]. ADA3 is critical in the recruitment of histone acetylases to promoter elements required for the expression of tumor suppressor genes [120]. E6 binding to hADA3 therefore may result in the absence of these tumor suppressors. Two-hybrid analyses also revealed the existence of another E6 protein partner, tuberin, a TSC2 tumor suppressor gene product. Tuberin is a component of the molecular complex that negatively controls cell growth and proliferation [114]. E6 binding to tuberin removes this negative regulation, thereby permitting cell growth and viral dissemination. Additionally, E6 influences cell cycle progression by binding to the $\mathrm{p} 53$ co-activator $\mathrm{CBP} / \mathrm{p} 300$, resulting in decreased affinity of p53 for its DNA binding site [121]. Yeast two-hybrid data reveals that HR-E6 binds to Gps2 and targets it for degradation. Gps2 interacts with $\mathrm{CBP} / \mathrm{p} 300$ and facilitates its transcriptional regulation of p53 [27, 92]; therefore E6 binding to both $\mathrm{CBP} / \mathrm{p} 300$ and Gps2 allows for the inappropriate survival and division of infected cells. Interactions of E6 with this set of proteins thus enhance the proliferation of virus-infected cells and cumulatively add to the oncogenic potential of HR-HPVs.

\section{Mediation of immune recognition}

To further facilitate virus survival, E6 interferes with the ability of the immune system to recognize infected cells and to encourage their elimination. Yeast two-hybrid experiments demonstrate that E6 interacts with interferon regulatory factor-3 (IRF-3). IRF-3 normally mediates the progression of apoptosis once a virus infection has been detected by the immune system. E6 binding obstructs the transactivation function of IRF-3, making it unable to properly regulate the cell death pathway $[17,96]$. Further, toll-like receptors (TLRs) are important components of the innate immune system. They primarily recognize the pathogen-associated molecular patterns displayed by various microbes and use them to generate signals that result in the elimination of the pathogen by the host immune system. Recently, it has been reported that E6 blocks the transcription of the TLR9 gene in the cervical cancer cell lines CaSki, SiHa, and HeLa [47]. This renders virusinfected cells unable to undergo apoptosis, giving rise to viral persistence in the host. These interactions of E6 with its respective protein partners inhibit apoptosis and facilitate viral propagation, and may therefore contribute to HPV-induced tumorigenesis.

Mediation of chromosomal stability

E6 has been shown to bind to the human minichromosome maintenance seven protein, hMCM7, leading to chromosomal abnormalities in E6-expressing cells [61]. Association of the MCM complex with chromatin normally 
leads to the onset of eukaryotic genome replication. E6 inhibits this association by mediating MCM7 degradation [77]. Loss of proper MCM7 function leads to the emergence of chromosomal abnormalities in infected cells which can contribute to carcinogenesis. Additionally, E6 interacts with and destabilizes O(6)-methylguanine DNA methyltransferase and XRCC1, proteins involved in singlestrand DNA break repair [54, 103]. Binding of HR-E6 to MCM7 leads to the development of chromosomal abnormalities which promote DNA damage. E6 interactions with O(6)-methylguanine DNA methyltransferase and XRCC1 obstruct the ability of these proteins to repair the damages incurred, giving rise to genomic instability in cells infected with HPV. Thus, binding of E6 to these proteins cooperatively contributes to viral genome replication and viral persistence. To avoid possible limitations in the proliferative capacity of these infected cells, HR-E6 increases hTERT gene transcription, a telomerase-multi-subunit complex that is responsible for synthesizing and adding telomeric sequences to the ends of chromosomes, thereby preventing DNA loss [59, 88, 93].

\section{Mediators of epithelial organization and differentiation}

Initial infection of HR-HPVs occurs in keratinocytes in the basal layer of squamous epithelium. Following integration of the viral genome into the host genome, viral DNA replication takes place as the infected cells undergo differentiation and cell division. Expression of the early oncogenes allows the virus to successfully replicate in the differentiated layers of the epithelium while also preventing the epithelial cells from undergoing terminal differentiation. Since cell proliferation often requires adhesion to an extracellular matrix (ECM), which would hamper dysplasia, HR-HPVs have developed ways in which to circumvent this problem.

Preservation of proper epithelial structure is important for a cell to appropriately regulate its growth. Normal cell division takes place when cells respond to proliferation signals and terminate when the signal is removed or when proper tissue size is reached. To overcome limitations in cell proliferation, HR-E6 interacts with and subsequently inactivates various proteins involved in epithelial organization. Paxillin, a protein that is associated with focal adhesion proteins and is implicated in actin organization, and thus in the maintenance of the cell cytoskeleton, has been shown to bind HR-E6 [113]. Binding of E6 to paxillin disrupts the formation of actin fibers, and it is this loss of epithelial integrity that supports viral transformation. Another key player in the promotion of cellular adhesion to the extracellular matrix (ECM) is zyxin. Zyxin is a focal adhesion molecule responsible for connecting the ECM to the cytoskeleton, for regulating cell proliferation and differentiation, for transmitting signals from the sites of cell adhesion to the nucleus, and for organizing actin, along with paxillin [26]. E6 binding to zyxin, in a manner similar to paxillin, impairs the ability of these proteins to maintain proper cell structure, which can lead to cellular transformation. Interactions between E6 and fibulin-1 have also been reported to support cellular transformation and tumor invasion by further contributing to the destabilization of the ECM [31]. In addition, E6 and the calcium-binding protein ERC-55 or E6-BP interact, resulting in the inability of epithelial cells to undergo terminal differentiation [18]. Together, these activities reduce the ability of the infected cell to adhere to the extracellular matrix and to sustain normal cell morphology, and enhance its replicative potential, thereby supporting cellular transformation and the potential development of oncogenesis.

Mediators of cell-cell adhesion, polarity, and proliferation control

The transformation and tumorigenic potential of HR-HPV has been linked to the ability of the oncoprotein E6 to bind additional proteins involved in mediating cell-cell adhesion, polarity, and proliferation control. hScrib, the human homolog of the Drosophila Scribble protein, behaves as a tumor suppressor by negatively regulating cell growth. E6 has been reported to bind to hScrib and to mediate its degradation, thus eliminating its control on cell cycle progression [83]. hScrib is primarily found associated with epithelial tight junctions, and loss of this protein leads to the inability of cells to adhere to the ECM. In addition, hDlg, the human homolog of the Drosophila disc large tumor suppressor, is required for cell-cell adhesion, apicobasal polarity, and epithelial cell proliferation. HR-E6 binding to hDlg not only disrupts proper formation of cellcell junctions, but also leads to loss of cell polarity and cell proliferation control [58, 70]. To further promote virus survival, HR-E6 binds to and mediates the degradation of PATJ [66, 105], which, like hScrib and hDlg, is important in the proper formation of epithelial tight junctions in polarized cells. HR-E6 also mediates the degradation of PTPN3, a membrane-associated tyrosine phosphatase involved in the phosphorylation of growth factor receptors [56]. In this way, degradation of PTPN3 allows HPVinfected cells to survive with less growth factor requirements. Together, binding of HR-E6 to these proteins gives rise to the aberrant morphology characteristic of cancer cells and supports the invasive growth of infected keratinocytes.

High-risk strains of human papillomavirus have also been shown to bind to other proteins that localize to the 
epithelial tight junctions, namely, MAGI-1, MAGI-2, and MAGI-3 [29]. In addition, the MAGI-2 and MAGI-3 proteins contribute to the regulation of the tumor suppressor PTEN. Mutations in PTEN are commonly associated with renal, breast, uterine, and advanced prostate cancers, suggesting that PTEN may serve as a marker of tumorigenicity for many cancers $[16,75]$. MUPP1, like hScrib and hDlg, plays a role in the negative regulation of cellular proliferation, and loss of its activity through E6 binding encourages cell cycle progression and cell growth. The MAGI proteins, along with the MUPP1 protein, are also thought to play a role in signal transduction, and E6mediated inhibition of their activity may disturb the proper formation of signaling complexes at the epithelial cell membranes [77]. The ability of HR-HPVs to efficiently transform cells and induce cellular proliferation, therefore, relies in part on the ability of E6 to bind and inactivate these numerous cellular proteins.

\section{E6 binding motifs: domains that direct oncoprotein binding}

E6 binds to a number of cellular proteins that mediate diverse activities in the cell, ranging from the regulation of apoptosis to the maintenance of chromosomal stability. Several attempts have been made to identify an E6 binding motif or a sequence to which E6 preferentially binds to its protein partners. In vitro and in vivo data demonstrate that binding of E6 to p53, Bak, hMCM7, E6TP1, and c-myc is facilitated by the E3 ubiquitin ligase moiety, E6-AP
[77, 78]. E6-AP is a $100-\mathrm{kDa}$ cellular protein that, upon interaction with E6, forms the complex necessary to mediate the ubiquitination and subsequent proteasomemediated degradation of these proteins. Data from yeast two-hybrid experiments incorporating a peptide library to identify peptides that associate with E6 [32], data from sequence comparisons of the known E6 binding partners [7] and data from the use of inhibitory peptides to specifically block E6 binding to these protein partners [72, 104] reveal that E6 binds to a consensus sequence characterized by the residues $\operatorname{Lxx} \phi \mathrm{Lsh}$ [5] in which $\mathrm{x}$ represents any amino acid, $\phi$ is a hydrophobic residue, $\mathrm{s}$ is an amino acid with a small side chain, and $\mathrm{h}$ is an amino acid that can make multiple hydrogen bonding interactions with its side chain (Fig. 2). In the case of E6/E6-AP interaction, the seven-residue leucine-containing motif, LQELLGE, mediates oncoprotein binding. This consensus sequence has also been referred to as an LXXLL motif [20, 21] to highlight the importance of the three conserved leucines at positions 9, 12, and 13 in facilitating E6 binding to its cellular partners, as substitution of these leucines has been reported to abolish E6/E6-AP interaction [7]. This same consensus sequence in the E6-BP/ERC-55, IRF-3, paxillin, and tuberin proteins directs oncoprotein binding to this set of partners. Once E6 has bound to E6-AP, the complex can then bind to additional proteins and mediate their degradation. Proteins in this group include p53, Bak, hMCM7, E6TP1, and c-myc.

A comparison of the sequences of HR-HPVs reveals the presence of a conserved C-terminal domain made up of the residues $\mathrm{XT} / \mathrm{SXV}$, where $\mathrm{X}$ is any amino acid.
Fig. 2 HR-E6 interacts with its cellular binding proteins via two known binding motifs, namely, the Lxx $\phi$ Lsh motif (or LXXLL motif) and the PDZ domain. However, the absence of these motifs in the sequences of many of its protein partners suggests the existence of at least one other motif that mediates oncoprotein binding

\begin{tabular}{|lll|}
\hline Motif to which E6 binds & Cellular partners of E6 & References \\
\hline E6 binds directly to these proteins & E6-AP & $32,53,72$ \\
by way of the Lxx $\phi$ Lsh (or LXXLL) & E6-BP/ERC-55 & 32 \\
motif & IRF-3 & 96 \\
& paxillin & 19,113 \\
& tuberin & 5 \\
\hline After E6 binds to E6-AP, the & Bak & 110 \\
E6/E6-AP complex binds to & c-myc & 57 \\
these proteins & E6TP1 & 38 \\
& hMCM7 & 60 \\
& p53 & $81,84,104$ \\
\hline \multirow{2}{*}{ PDZ domain } & hScrib & 107 \\
& hDlg & 45 \\
& MAGI & 85 \\
& MUPP1 & 66 \\
& PATJ & $8,66,79$ \\
& PTPN3 & 56 \\
\hline Unknown E6 binding motif & FADD & 35 \\
& Gps2 & 27 \\
& hADA3 & 62 \\
& procaspase 8 & 36 \\
\hline
\end{tabular}


Interestingly, this sequence of amino acids is lacking in LR strains of HPV [58]. This abbreviated stretch of residues directs oncoprotein binding to the hDlg, hScrib, MUPP1, PATJ, PTPN3, and MAGI proteins. The interaction of E6 with these proteins is mediated not by the seven-residue leucine-containing motif, however, but by a conserved PDZ domain, a region of $\sim 90$ amino acids first recognized in the sequence of a number of signaling molecules. PDZcontaining proteins are often involved in signal transduction, transcriptional regulation, and cell polarity. Mice expressing a version of E6 which lacks the C-terminal five amino acids that mediate binding to the PDZ domain render these mice unable to trigger unregulated cellular proliferation. This finding highlights the importance of the PDZ domain in directing E6-induced hyperplasia [39, 69, 85]. Therefore, to date, two E6-binding motifs have been identified (Fig. 2).

Interestingly, several of the known binding partners of E6, including FADD, Gps2, hADA3 and procaspase 8, are lacking both the LXXLL motif and the PDZ domain. This suggests the existence of at least one additional binding motif for the oncoprotein.

\section{Inhibitors of E6 binding and therapeutic potential}

At present, two vaccines have been developed for the prevention of HPV-induced cervical cancer: a quadrivalent vaccine, Gardasil ${ }^{\circledR}$, manufactured by Merck \& Co., intended to prevent infection with the HPV strains 6, 11, 16, and 18 , and a bivalent vaccine, Cervarix ${ }^{\circledR}$, manufactured by GlaxoSmithKline, intended to prevent infection with strains 16 and 18. These prophylactic vaccines are designed to induce the generation of antibodies against the L1 and L2 capsid proteins of HPV in the hopes that these antibodies will neutralize future virus infection. These vaccines, if administered before exposure to HPV, are reported to be highly effective at protecting women from infection [3, 106]. However, they are not therapeutic, and papillomavirus infections often remain undetectable for prolonged periods of time. Therefore, therapeutic vaccines, designed to target the early non-structural proteins of the virus, such as E6, may be more effective once infection has occurred.

Successful infection with HR-HPV is usually accompanied by persistent expression of the E6 oncoprotein [22, 97]. Interactions between E6 and its many cellular binding partners contribute to viral propagation and the possible progression to cancer. These characteristics have therefore fueled the development of possible therapeutic agents to treat already established HPV infections. Various types of therapeutic vaccines are being developed that target the early proteins of HR-HPV, especially the oncoproteins E6 and E7, since these are continuously expressed in cervical tumors. The vaccines that have been generated include those that are viral/bacterial based [13, 49], peptide based [94], protein based [25, 63], or DNA based [91]. If successful, these therapeutic vaccines have the potential to treat HPV-associated premalignant tumors. Unfortunately, such vaccines are expected to have numerous limitations, including the loss of vaccine effectiveness in individuals with a pre-existing viral immunity, safety concerns when administering viral/bacterial based vaccines, and the weak immunogenicity of peptide and protein-based vaccines [76].

Therefore, a more in-depth understanding of the molecular interactions of E6 that lead to oncogenesis is being pursued, which will allow for the development of additional approaches to either inhibit E6 expression or combat the consequences that arise from E6 activity. For example, the literature describes experiments to block expression of E6 in HPV-positive cancer cells by inhibiting viral transcription [43], by administering anti-sense constructs [46], and by administering RNAi targeting E6 [115] in cell culture. The results obtained indicate that these approaches can lead to an absence of detectable transcript, an increase in the tumor suppressor p53 and a concomitant increase in the induction of apoptosis, characteristics of a more normal phenotype.

Once transcription has taken place and the E6 protein is expressed, it has been shown that antibodies specifically designed to target particular regions of E6 can be successfully used to obstruct E6 activity [44]. Co-immunoprecipitation assays demonstrate that antibodies generated to recognize epitopes in the N-terminus of E6 blocked E6 association with p53. Similarly, antibodies generated to recognize the second zinc finger domain on E6, which is required for E6-AP binding, blocked E6/E6AP association and prevented E6-mediated p53 degradation [64]. Monoclonal antibodies to the N-terminal ten amino acids of E6 were shown to specifically bind to the oncoprotein and inhibit proteolysis of p53 in HPV-16positive CaSki cells, as shown by immunoblot [42]. Peptide aptamers, molecules designed to selectively bind to a given target protein and block its activity in vivo, have been effectively used to inhibit E6 binding to its respective partners, resulting in increased p53 levels and the apoptotic elimination of HPV-positive SiHa cells [15].

The identification of a conserved binding domain within HPV 16 E6 binding partners, the LXXLL motif, has led to the development of peptide inhibitors that harbor this motif $[11,15]$. Additionally, knowledge of the amino acids that constitute the $\alpha$-helical E6-binding motif has allowed for the screening of chemical compounds harboring functional groups necessary for binding E6. These compounds would thus serve as chemical inhibitors of E6 binding to its target 
proteins in cells. Administration of these inhibitors has been reported to competitively inhibit binding of E6 to its natural partners, E6-AP and E6-BP, and thus interfere with the ability of E6 to promote p53 degradation [5].

Interactions of E6 with PDZ-domain-containing proteins are also important in facilitating the malignant progression of HPV-infected cells. The residues on E6 necessary to bind the PDZ domain localize to a small, exposed region on the surface of the protein, suggesting that therapeutic agents designed to block these interactions may be feasible [112]. Moreover, knowledge of the amino acid sequences that direct binding of HR-E6 to its protein partners further contributes to the potential of developing agents that block E6 binding to its cellular targets, resulting in the possible re-sensitization of HPV-infected cells to normal cell-deathinducing stimuli.

\section{Conclusions}

To survive and propagate in their host after infection, viruses have developed varying strategies to avoid immune clearance. Many viruses are able to manipulate key components of the cell death pathway. These viruses can produce homologs of cytokines, chemokines, or their receptors that competitively bind to their respective cellular partners, thereby preventing the generation of an apoptotic signal [2, 9]. Members of the poxvirus family produce soluble decoy receptors that obstruct the initial ligand-receptor interaction at the cell membrane. The cowpox virus, for instance, produces the TNFR-2 ortho$\operatorname{logs}, \mathrm{CrmB}, \mathrm{CrmC}, \mathrm{CrmD}$, and $\mathrm{CrmE}$ that bind to TNF and inhibit initiation of apoptosis [101]. Further downstream, the bovine herpesvirus 4 (BHV4) produces the BORFE2 protein intended to interact with the death effector domain of caspase 8 and prevent the propagation of the apoptotic signal from the Fas- or TNFR pathway [116]. Similarly, the molluscum contagiosum virus inhibits Fas-induced apoptosis when its MC159 protein binds to cellular FADD [10, 102]. HPV also engages mechanisms designed to escape elimination by the host. The E6 and E7 oncoproteins of HPV interact with either the tumor suppressor protein p53 [122] or pRb [71], respectively, and in doing so, allow for cell cycle progression as well as resistance to apoptotic signals. It has also been reported that E6 binds to the death effector domains of FADD and procaspase 8 and that binding leads to E6-mediated degradation of these proteins and the resultant failure of HPV-expressing cells to undergo apoptosis [35, 36]. Many different viruses have developed mechanisms by which to disrupt the extrinsic arm of apoptosis, suggesting that inactivating this cell death pathway is integral for virus infection and survival.
High-risk strains of human papillomavirus are the causative agents of cervical cancer and have recently been found to be associated with $\sim 20 \%$ of cancers of the oropharynx [51]. The HPV E6 protein is a fundamental contributor to the oncogenic potential of the virus, and it is its interactions with various cellular proteins that enable the virus to persist. During the course of HPV infection, E6 binds and inactivates a number of key regulators of cell cycle progression in order to permit infected cells to proliferate. HR-E6 also blocks apoptosis by targeting p53, Bak, c-myc, FADD and procaspase 8 for degradation. Additionally, binding of E6 to E6TP1, hADA3, tuberin, $\mathrm{CBP} / \mathrm{p} 300$, and Gps2 hampers the ability of these proteins to negatively regulate cell proliferation. HPV is also able to suppress the innate immune system, through the binding of E6 to IRF-3 and the E6-mediated down-regulation of TLR9 expression, making the immune system unable to recognize and clear viral infection. Taken together, these interactions collectively result in the deregulated multiplicity of virusinfected cells.

Cancer cells are often associated with chromosomal instability, DNA damage and a loss in the structural integrity of epithelial cells. These factors allow cancer cells to thwart the normal restrictions of cell division, thus stimulating immortalization. E6 binding to hMCM7 promotes chromosomal instability, while binding to $\mathrm{O}(6)$-methylguanine DNA methyltransferase and XRCC1 prevents the repair of the DNA damage that arises from this instability. To prevent possible limitations to cell cycle progression, E6 stabilizes hTERT and up-regulates its ability to catalyze the synthesis and addition of telomeres to the ends of chromosomes. Heightened telomerase activity allows for limitless proliferative capacity of HPVinfected cells. In addition to these proteins, E6 also binds to a specific group of PDZ-domain-containing proteins, including hScrib, hDlg, MAGI-family proteins, MUPP1, PATJ, and PTPN3. In this way, HR-E6 impairs the generation of appropriate cell-cell adhesion, polarity, and proliferation control. Improper formation of focal adhesions to the ECM, loss of proper cell polarity, loss of cell cycle regulation, and the up-regulation of virus-infected cell proliferation cumulatively contribute to the progression to malignancy.

Discovery of the numerous cellular binding partners of HR-E6 has allowed for the identification of two binding motifs or sequences which E6 preferably targets for interaction, the seven-residue leucine-containing motif, $\operatorname{Lxx} \phi$ Lsh, and the PDZ domain. As mentioned previously, these domains are absent in the sequences of FADD, Gps2, hADA3, and procaspase 8, although binding of E6 to these proteins has been shown. This suggests the existence of at least one additional E6-binding motif. Current and future studies on E6 and its cellular targets have significant 
implications for the development of possible therapeutic approaches for cervical cancer as well as HPV-associated head and neck cancers. The potential utilization of a multipronged approach, including both prophylactic and therapeutic vaccines as well as molecular approaches to prevent and treat HPV-positive cancers makes this an exciting time for HPV-related studies.

Acknowledgments I would like to acknowledge my NIH grant: R01 CA095461 from the National Cancer Institute.

Open Access This article is distributed under the terms of the Creative Commons Attribution Noncommercial License which permits any noncommercial use, distribution, and reproduction in any medium, provided the original author(s) and source are credited.

\section{References}

1. Alani RM, Munger K (1998) Human papillomaviruses and associated malignancies. J Clin Oncol 16:330-337

2. Alcami A (2003) Viral mimicry of cytokines, chemokines and their receptors. Nat Rev Immunol 3:36-50

3. Ames A, Gravitt P (2007) Human papillomavirus vaccine update. Curr Infect Dis Rep 9:151-158

4. Arbeit JM, Munger K, Howley PM, Hanahan D (1994) Progressive squamous epithelial neoplasia in K14-human papillomavirus type 16 transgenic mice. J Virol 68:4358-4368

5. Baleja JD, Cherry JJ, Liu Z, Gao H, Nicklaus MC, Voigt JH, Chen JJ, Androphy EJ (2006) Identification of inhibitors to papillomavirus type 16 E6 protein based on three-dimensional structures of interacting proteins. Antiviral Res 72:49-59

6. Band V, De Caprio JA, Delmolino L, Kulesa V, Sager R (1991) Loss of p53 protein in human papillomavirus type 16 E6immortalized human mammary epithelial cells. J Virol 65:66716676

7. Be X, Hong Y, Wei J, Androphy EJ, Chen JJ, Baleja JD (2001) Solution structure determination and mutational analysis of the papillomavirus E6 interacting peptide of E6AP. Biochemistry 40:1293-1299

8. Bedell MA, Jones KH, Laimins LA (1987) The E6-E7 region of human papillomavirus type 18 is sufficient for transformation of NIH 3T3 and rat-1 cells. J Virol 61:3635-3640

9. Benedict CA (2003) Viruses and the TNF-related cytokines, an evolving battle. Cytokine Growth Factor Rev 14:349-357

10. Bertin J, Armstrong RC, Ottilie S, Martin DA, Wang Y, Banks S, Wang GH, Senkevich TG, Alnemri ES, Moss B, Lenardo MJ, Tomaselli KJ, Cohen JI (1997) Death effector domain-containing herpesvirus and poxvirus proteins inhibit both Fas- and TNFR1-induced apoptosis. Proc Natl Acad Sci USA 94:11721176

11. Bohl J, Das K, Dasgupta B, Vande Pol SB (2000) Competitive binding to a charged leucine motif represses transformation by a papillomavirus E6 oncoprotein. Virology 271:163-170

12. Borbely AA, Murvai M, Konya J, Beck Z, Gergely L, Li F, Veress G (2006) Effects of human papillomavirus type 16 oncoproteins on survivin gene expression. J Gen Virol 87:287294

13. Borysiewicz LK, Fiander A, Nimako M, Man S, Wilkinson GW, Westmoreland D, Evans AS, Adams M, Stacey SN, Boursnell ME, Rutherford E, Hickling JK, Inglis SC (1996) A recombinant vaccinia virus encoding human papillomavirus types 16 and 18 ,
E6 and E7 proteins as immunotherapy for cervical cancer. Lancet 347:1523-1527

14. Brink AA, Zielinski GD, Steenbergen RD, Snijders PJ, Meijer CJ (2005) Clinical relevance of human papillomavirus testing in cytopathology. Cytopathology 16:7-12

15. Butz K, Denk C, Ullmann A, Scheffner M, Hoppe-Seyler F (2000) Induction of apoptosis in human papillomaviruspositive cancer cells by peptide aptamers targeting the viral E6 oncoprotein. Proc Natl Acad Sci USA 97:6693-6697

16. Cairns P, Okami K, Halachmi S, Halachmi N, Esteller M, Herman JG, Jen J, Isaacs WB, Bova GS, Sidransky D (1997) Frequent inactivation of PTEN/MMAC1 in primary prostate cancer. Cancer Res 57:4997-5000

17. Chakrabarti O, Krishna S (2003) Molecular interactions of 'high risk' human papillomaviruses E6 and E7 oncoproteins: implications for tumour progression. J Biosci 28:337-348

18. Chen JJ, Reid CE, Band V, Androphy EJ (1995) Interaction of papillomavirus E6 oncoproteins with a putative calcium-binding protein. Science 269:529-531

19. Chen JJ, Hong Y, Rustamzadeh E, Baleja JD, Androphy EJ (1998) Identification of an alpha helical motif sufficient for association with papillomavirus E6. J Biol Chem 273:13537-13544

20. Cooper B, Schneider S, Bohl J, Jiang Y, Beaudet A, Vande Pol S (2003) Requirement of E6AP and the features of human papillomavirus E6 necessary to support degradation of p53. Virology 306:87-99

21. Cooper B, Brimer N, Vande Pol SB (2007) Human Papillomavirus E6 regulates the cytoskeleton dynamics of keratinocytes through targeted degradation of p53. J Virol 81:12675-12679

22. Cripe TP, Haugen TH, Turk JP, Tabatabai F, Schmid PG 3rd, Durst M, Gissmann L, Roman A, Turek LP (1987) Transcriptional regulation of the human papillomavirus-16 E6-E7 promoter by a keratinocyte-dependent enhancer, and by viral E2 trans-activator and repressor gene products: implications for cervical carcinogenesis. Embo J 6:3745-3753

23. Crook T, Tidy JA, Vousden KH (1991) Degradation of p53 can be targeted by HPV E6 sequences distinct from those required for p53 binding and trans-activation. Cell 67:547-556

24. Dalal S, Gao Q, Androphy EJ, Band V (1996) Mutational analysis of human papillomavirus type 16 E6 demonstrates that p53 degradation is necessary for immortalization of mammary epithelial cells. J Virol 70:683-688

25. de Jong A, O'Neill T, Khan AY, Kwappenberg KM, Chisholm SE, Whittle NR, Dobson JA, Jack LC, St Clair Roberts JA, Offringa R, van der Burg SH, Hickling JK (2002) Enhancement of human papillomavirus (HPV) type $16 \mathrm{E} 6$ and E7-specific Tcell immunity in healthy volunteers through vaccination with TA-CIN, an HPV16 L2E7E6 fusion protein vaccine. Vaccine 20:3456-3464

26. Degenhardt YY, Silverstein S (2001) Interaction of zyxin, a focal adhesion protein, with the e6 protein from human papillomavirus type 6 results in its nuclear translocation. J Virol 75:11791-11802

27. Degenhardt YY, Silverstein SJ (2001) Gps2, a protein partner for human papillomavirus E6 proteins. J Virol 75:151-160

28. Dell G, Gaston K (2001) Human papillomaviruses and their role in cervical cancer. Cell Mol Life Sci 58:1923-1942

29. Dobrosotskaya I, Guy RK, James GL (1997) MAGI-1, a membrane-associated guanylate kinase with a unique arrangement of protein-protein interaction domains. J Biol Chem 272:3158931597

30. Doorbar J (2006) Molecular biology of human papillomavirus infection and cervical cancer. Clin Sci (Lond) 110:525-541

31. Du M, Fan X, Hong E, Chen JJ (2002) Interaction of oncogenic papillomavirus E6 proteins with fibulin-1. Biochem Biophys Res Commun 296:962-969 
32. Elston RC, Napthine S, Doorbar J (1998) The identification of a conserved binding motif within human papillomavirus type 16 E6 binding peptides, E6AP and E6BP. J Gen Virol 79(Pt 2):371-374

33. Felsani A, Mileo AM, Paggi MG (2006) Retinoblastoma family proteins as key targets of the small DNA virus oncoproteins. Oncogene 25:5277-5285

34. Filippova M, Song H, Connolly JL, Dermody TS, DuerksenHughes PJ (2002) The human papillomavirus 16 E6 protein binds to tumor necrosis factor (TNF) R1 and protects cells from TNF-induced apoptosis. J Biol Chem 277:21730-21739

35. Filippova M, Parkhurst L, Duerksen-Hughes PJ (2004) The human papillomavirus 16 E6 protein binds to Fas-associated death domain and protects cells from Fas-triggered apoptosis. J Biol Chem 279:25729-25744

36. Filippova M, Johnson MM, Bautista M, Filippov V, Fodor N, Tungteakkhun SS, Williams K, Duerksen-Hughes PJ (2007) The large and small isoforms of human papillomavirus type 16 E6 bind to and differentially affect procaspase 8 stability and activity. J Virol 81:4116-4129

37. Gao Q, Srinivasan S, Boyer SN, Wazer DE, Band V (1999) The E6 oncoproteins of high-risk papillomaviruses bind to a novel putative GAP protein, E6TP1, and target it for degradation. Mol Cell Biol 19:733-744

38. Gao Q, Kumar A, Singh L, Huibregtse JM, Beaudenon S, Srinivasan S, Wazer DE, Band H, Band V (2002) Human papillomavirus E6-induced degradation of E6TP1 is mediated by E6AP ubiquitin ligase. Cancer Res 62:3315-3321

39. Gardiol D, Galizzi S, Banks L (2002) Mutational analysis of the discs large tumour suppressor identifies domains responsible for human papillomavirus type 18 E6-mediated degradation. J Gen Virol 83:283-289

40. Garland SM (2002) Human papillomavirus update with a particular focus on cervical disease. Pathology 34:213-224

41. Gillison ML, Koch WM, Capone RB, Spafford M, Westra WH, Wu L, Zahurak ML, Daniel RW, Viglione M, Symer DE, Shah KV, Sidransky D (2000) Evidence for a causal association between human papillomavirus and a subset of head and neck cancers. J Natl Cancer Inst 92:709-720

42. Giovane C, Trave G, Briones A, Lutz Y, Wasylyk B, Weiss E (1999) Targetting of the N-terminal domain of the human papillomavirus type 16 E6 oncoprotein with monomeric ScFvs blocks the E6-mediated degradation of cellular p53. J Mol Recognit 12:141-152

43. Goodwin EC, DiMaio D (2000) Repression of human papillomavirus oncogenes in HeLa cervical carcinoma cells causes the orderly reactivation of dormant tumor suppressor pathways. Proc Natl Acad Sci USAm 97:12513-12518

44. Griffin H, Elston R, Jackson D, Ansell K, Coleman M, Winter G, Doorbar J (2006) Inhibition of papillomavirus protein function in cervical cancer cells by intrabody targeting. J Mol Biol 355:360-378

45. Grm HS, Banks L (2004) Degradation of hDlg and MAGIs by human papillomavirus E6 is E6AP-independent. J Gen Virol 85:2815-2819

46. Hamada K, Sakaue M, Alemany R, Zhang WW, Horio Y, Roth JA, Mitchell MF (1996) Adenovirus-mediated transfer of HPV 16 E6/E7 antisense RNA to human cervical cancer cells. Gynecol Oncol 63:219-227

47. Hasan UA, Bates E, Takeshita F, Biliato A, Accardi R, Bouvard V, Mansour M, Vincent I, Gissmann L, Iftner T, Sideri M, Stubenrauch F, Tommasino M (2007) TLR9 expression and function is abolished by the cervical cancer-associated human papillomavirus type 16. J Immunol 178:3186-3197

48. Hawley-Nelson P, Vousden KH, Hubbert NL, Lowy DR, Schiller JT (1989) HPV16 E6 and E7 proteins cooperate to immortalize human foreskin keratinocytes. Embo J 8:39053910

49. He Z, Wlazlo AP, Kowalczyk DW, Cheng J, Xiang ZQ, GilesDavis W, Ertl HC (2000) Viral recombinant vaccines to the E6 and E7 antigens of HPV-16. Virology 270:146-161

50. Hebner CM, Laimins LA (2006) Human papillomaviruses: basic mechanisms of pathogenesis and oncogenicity. Rev Med Virol 16:83-97

51. Herrero R, Castellsague X, Pawlita M, Lissowska J, Kee F, Balaram P, Rajkumar T, Sridhar H, Rose B, Pintos J, Fernandez L, Idris A, Sanchez MJ, Nieto A, Talamini R, Tavani A, Bosch FX, Reidel U, Snijders PJ, Meijer CJ, Viscidi R, Munoz N, Franceschi S (2003) Human papillomavirus and oral cancer: the international agency for research on cancer multicenter study. J Natl Cancer Inst 95:1772-1783

52. Horiuchi J, Silverman N, Marcus GA, Guarente L (1995) ADA3, a putative transcriptional adaptor, consists of two separable domains and interacts with ADA2 and GCN5 in a trimeric complex. Mol Cell Biol 15:1203-1209

53. Huibregtse JM, Scheffner M, Howley PM (1993) Localization of the E6-AP regions that direct human papillomavirus E6 binding, association with $\mathrm{p} 53$, and ubiquitination of associated proteins. Mol Cell Biol 13:4918-4927

54. Iftner T, Elbel M, Schopp B, Hiller T, Loizou JI, Caldecott KW, Stubenrauch F (2002) Interference of papillomavirus E6 protein with single-strand break repair by interaction with XRCC1. Embo J 21:4741-4748

55. Jemal A, Tiwari RC, Murray T, Ghafoor A, Samuels A, Ward E, Feuer EJ, Thun MJ (2004) Cancer statistics, 2004. CA Cancer J Clin 54:8-29

56. Jing M, Bohl J, Brimer N, Kinter M, Vande Pol SB (2007) Degradation of tyrosine phosphatase PTPN3 (PTPH1) by association with oncogenic human papillomavirus E6 proteins. J Virol 81:2231-2239

57. Kinoshita T, Shirasawa H, Shino Y, Moriya H, Desbarats L, Eilers M, Simizu B (1997) Transactivation of prothymosin alpha and c-myc promoters by human papillomavirus type 16 E6 protein. Virology 232:53-61

58. Kiyono T, Hiraiwa A, Fujita M, Hayashi Y, Akiyama T, Ishibashi M (1997) Binding of high-risk human papillomavirus E6 oncoproteins to the human homologue of the Drosophila discs large tumor suppressor protein. Proc Natl Acad Sci USA 94:11612-11616

59. Kiyono T, Foster SA, Koop JI, McDougall JK, Galloway DA, Klingelhutz AJ (1998) Both Rb/p16INK4a inactivation and telomerase activity are required to immortalize human epithelial cells. Nature 396:84-88

60. Kuhne C, Gardiol D, Guarnaccia C, Amenitsch H, Banks L (2000) Differential regulation of human papillomavirus E6 by protein kinase A: conditional degradation of human discs large protein by oncogenic E6. Oncogene 19:5884-5891

61. Kukimoto I, Aihara S, Yoshiike K, Kanda T (1998) Human papillomavirus oncoprotein E6 binds to the $\mathrm{C}$-terminal region of human minichromosome maintenance 7 protein. Biochem Biophys Res Commun 249:258-262

62. Kumar A, Zhao Y, Meng G, Zeng M, Srinivasan S, Delmolino LM, Gao Q, Dimri G, Weber GF, Wazer DE, Band H, Band V (2002) Human papillomavirus oncoprotein E6 inactivates the transcriptional coactivator human ADA3. Mol Cell Biol 22:5801-5812

63. Lacey CJ, Thompson HS, Monteiro EF, O'Neill T, Davies ML, Holding FP, Fallon RE, Roberts JS (1999) Phase IIa safety and immunogenicity of a therapeutic vaccine, TA-GW, in persons with genital warts. J Infect Dis 179:612-618

64. Lagrange M, Charbonnier S, Orfanoudakis G, Robinson P, Zanier K, Masson M, Lutz Y, Trave G, Weiss E, Deryckere F 
(2005) Binding of human papillomavirus 16 E6 to p53 and E6AP is impaired by monoclonal antibodies directed against the second zinc-binding domain of E6. J Gen Virol 86:1001-1007

65. Laimins LA (1993) The biology of human papillomaviruses: from warts to cancer. Infect Agents Dis 2:74-86

66. Latorre IJ, Roh MH, Frese KK, Weiss RS, Margolis B, Javier RT (2005) Viral oncoprotein-induced mislocalization of select PDZ proteins disrupts tight junctions and causes polarity defects in epithelial cells. J Cell Sci 118:4283-4293

67. Lechner MS, Laimins LA (1994) Inhibition of p53 DNA binding by human papillomavirus E6 proteins. J Virol 68:4262-4273

68. Ledwaba T, Dlamini Z, Naicker S, Bhoola K (2004) Molecular genetics of human cervical cancer: role of papillomavirus and the apoptotic cascade. Biol Chem 385:671-682

69. Lee C, Laimins LA (2004) Role of the PDZ domain-binding motif of the oncoprotein E6 in the pathogenesis of human papillomavirus type 31 . J Virol 78:12366-12377

70. Lee SS, Weiss RS, Javier RT (1997) Binding of human virus oncoproteins to hDlg/SAP97, a mammalian homolog of the Drosophila discs large tumor suppressor protein. Proc Natl Acad Sci USA 94:6670-6675

71. Liu X, Clements A, Zhao K, Marmorstein R (2006) Structure of the human Papillomavirus E7 oncoprotein and its mechanism for inactivation of the retinoblastoma tumor suppressor. J Biol Chem 281:578-586

72. Liu Y, Liu Z, Androphy E, Chen J, Baleja JD (2004) Design and characterization of helical peptides that inhibit the E6 protein of papillomavirus. Biochemistry 43:7421-7431

73. Longworth MS, Laimins LA (2004) Pathogenesis of human papillomaviruses in differentiating epithelia. Microbiol Mol Biol Rev 68:362-372

74. Lowy DR, Kirnbauer R, Schiller JT (1994) Genital human papillomavirus infection. Proc Natl Acad Sci USA 91:24362440

75. Lynch ED, Ostermeyer EA, Lee MK, Arena JF, Ji H, Dann J, Swisshelm K, Suchard D, MacLeod PM, Kvinnsland S, Gjertsen BT, Heimdal K, Lubs H, Moller P, King MC (1997) Inherited mutations in PTEN that are associated with breast cancer, cowden disease, and juvenile polyposis. Am J Hum Genet 61:1254-1260

76. Mahdavi A, Monk BJ (2005) Vaccines against human papillomavirus and cervical cancer: promises and challenges. Oncologist 10:528-538

77. Mantovani F, Banks L (2001) The human papillomavirus E6 protein and its contribution to malignant progression. Oncogene 20:7874-7887

78. Matsumoto Y, Nakagawa S, Yano T, Takizawa S, Nagasaka K, Nakagawa K, Minaguchi T, Wada O, Ooishi H, Matsumoto K, Yasugi T, Kanda T, Huibregtse JM, Taketani Y (2006) Involvement of a cellular ubiquitin-protein ligase E6AP in the ubiquitin-mediated degradation of extensive substrates of highrisk human papillomavirus E6. J Med Virol 78:501-507

79. Motoyama S, Ladines-Llave CA, Luis Villanueva S, Maruo T (2004) The role of human papilloma virus in the molecular biology of cervical carcinogenesis. Kobe J Med Sci 50:9-19

80. Munger K, Phelps WC, Bubb V, Howley PM, Schlegel R (1989) The E6 and E7 genes of the human papillomavirus type 16 together are necessary and sufficient for transformation of primary human keratinocytes. J Virol 63:4417-4421

81. Munger K, Howley PM (2002) Human papillomavirus immortalization and transformation functions. Virus Res 89:213-228

82. Munger K, Baldwin A, Edwards KM, Hayakawa H, Nguyen CL, Owens M, Grace M, Huh K (2004) Mechanisms of human papillomavirus-induced oncogenesis. J Virol 78:11451-11460

83. Nakagawa S, Huibregtse JM (2000) Human scribble (Vartul) is targeted for ubiquitin-mediated degradation by the high-risk papillomavirus E6 proteins and the E6AP ubiquitin-protein ligase. Mol Cell Biol 20:8244-8253

84. Narisawa-Saito M, Kiyono T (2007) Basic mechanisms of highrisk human papillomavirus-induced carcinogenesis: roles of E6 and E7 proteins. Cancer Sci 98(10):1505-1511

85. Nguyen ML, Nguyen MM, Lee D, Griep AE, Lambert PF (2003) The PDZ ligand domain of the human papillomavirus type 16 E6 protein is required for E6's induction of epithelial hyperplasia in vivo. J Virol 77:6957-6964

86. Nomine Y, Ristriani T, Laurent C, Lefevre JF, Weiss E, Trave G (2001) Formation of soluble inclusion bodies by hpv e6 oncoprotein fused to maltose-binding protein. Protein Expr Purif 23:22-32

87. Nomine Y, Masson M, Charbonnier S, Zanier K, Ristriani T, Deryckere F, Sibler AP, Desplancq D, Atkinson RA, Weiss E, Orfanoudakis G, Kieffer B, Trave G (2006) Structural and functional analysis of E6 oncoprotein: insights in the molecular pathways of human papillomavirus-mediated pathogenesis. Mol Cell 21:665-678

88. Oh ST, Kyo S, Laimins LA (2001) Telomerase activation by human papillomavirus type 16 E6 protein: induction of human telomerase reverse transcriptase expression through Myc and GC-rich Sp1 binding sites. J Virol 75:5559-5566

89. Pan H, Griep AE (1995) Temporally distinct patterns of p53dependent and p53-independent apoptosis during mouse lens development. Genes Dev 9:2157-2169

90. Parkin DM, Bray F, Ferlay J, Pisani P (2005) Global cancer statistics, 2002. CA Cancer J Clin 55:74-108

91. Peng S, Tomson TT, Trimble C, He L, Hung CF, Wu TC (2006) A combination of DNA vaccines targeting human papillomavirus type 16 E6 and E7 generates potent antitumor effects. Gene Ther 13:257-265

92. Peng YC, Breiding DE, Sverdrup F, Richard J, Androphy EJ (2000) AMF-1/Gps2 binds p300 and enhances its interaction with papillomavirus E2 proteins. J Virol 74:5872-5879

93. Rapp L, Chen JJ (1998) The papillomavirus E6 proteins. Biochim Biophys Acta 1378:F1-F19

94. Ressing ME, Sette A, Brandt RM, Ruppert J, Wentworth PA, Hartman M, Oseroff C, Grey HM, Melief CJ, Kast WM (1995) Human CTL epitopes encoded by human papillomavirus type 16 E6 and E7 identified through in vivo and in vitro immunogenicity studies of HLA-A*0201-binding peptides. J Immunol 154:5934-5943

95. Romanczuk H, Howley PM (1992) Disruption of either the E1 or the E2 regulatory gene of human papillomavirus type 16 increases viral immortalization capacity. Proc Natl Acad Sci USA 89:3159-3163

96. Ronco LV, Karpova AY, Vidal M, Howley PM (1998) Human papillomavirus $16 \mathrm{E} 6$ oncoprotein binds to interferon regulatory factor-3 and inhibits its transcriptional activity. Genes Dev 12:2061-2072

97. Scheffner M, Werness BA, Huibregtse JM, Levine AJ, Howley PM (1990) The E6 oncoprotein encoded by human papillomavirus types 16 and 18 promotes the degradation of p53. Cell 63:1129-1136

98. Scheffner M, Huibregtse JM, Vierstra RD, Howley PM (1993) The HPV-16 E6 and E6-AP complex functions as a ubiquitin-protein ligase in the ubiquitination of p53. Cell 75:495-505

99. Scheurer ME, Tortolero-Luna G, Adler-Storthz K (2005) Human papillomavirus infection: biology, epidemiology, and prevention. Int J Gynecol Cancer 15:727-746

100. Shai A, Brake T, Somoza C, Lambert PF (2007) The human papillomavirus E6 oncogene dysregulates the cell cycle and contributes to cervical carcinogenesis through two independent activities. Cancer Res 67:1626-1635 
101. Shisler J, Yang C, Walter B, Ware CF, Gooding LR (1997) The adenovirus E3-10.4K/14.5K complex mediates loss of cell surface Fas (CD95) and resistance to Fas-induced apoptosis. J Virol 71:8299-8306

102. Shisler JL, Moss B (2001) Molluscum contagiosum virus inhibitors of apoptosis: the MC159 v-FLIP protein blocks Fasinduced activation of procaspases and degradation of the related MC160 protein. Virology 282:14-25

103. Srivenugopal KS, Ali-Osman F (2002) The DNA repair protein, $\mathrm{O}(6)$-methylguanine-DNA methyltransferase is a proteolytic target for the E6 human papillomavirus oncoprotein. Oncogene 21:5940-5945

104. Sterlinko Grm H, Weber M, Elston R, McIntosh P, Griffin H, Banks L, Doorbar J (2004) Inhibition of E6-induced degradation of its cellular substrates by novel blocking peptides. J Mol Biol 335:971-985

105. Storrs CH, Silverstein SJ (2007) PATJ, a tight junction-associated PDZ protein, is a novel degradation target of high-risk human papillomavirus E6 and the alternatively spliced isoform 18 E6. J Virol 81:4080-4090

106. Szarewski A (2007) Prophylactic HPV vaccines. Eur J Gynaecol Oncol 28:165-169

107. Takizawa S, Nagasaka K, Nakagawa S, Yano T, Nakagawa K, Yasugi T, Takeuchi T, Kanda T, Huibregtse JM, Akiyama T, Taketani Y (2006) Human scribble, a novel tumor suppressor identified as a target of high-risk HPV E6 for ubiquitin-mediated degradation, interacts with adenomatous polyposis coli. Genes Cells 11:453-464

108. Talis AL, Huibregtse JM, Howley PM (1998) The role of E6AP in the regulation of p53 protein levels in human papillomavirus (HPV)-positive and HPV-negative cells. J Biol Chem 273:64396445

109. Thomas JT, Hubert WG, Ruesch MN, Laimins LA (1999) Human papillomavirus type 31 oncoproteins E6 and E7 are required for the maintenance of episomes during the viral life cycle in normal human keratinocytes. Proc Natl Acad Sci USA 96:8449-8454

110. Thomas M, Banks L (1999) Human papillomavirus (HPV) E6 interactions with Bak are conserved amongst E6 proteins from high and low risk HPV types. J Gen Virol 80(Pt 6):1513-1517

111. Thomas M, Laura R, Hepner K, Guccione E, Sawyers C, Lasky L, Banks L (2002) Oncogenic human papillomavirus E6 proteins target the MAGI-2 and MAGI-3 proteins for degradation. Oncogene 21:5088-5096
112. Tochio H, Hung F, Li M, Bredt DS, Zhang M (2000) Solution structure and backbone dynamics of the second PDZ domain of postsynaptic density-95. J Mol Biol 295:225-237

113. Tong X, Howley PM (1997) The bovine papillomavirus E6 oncoprotein interacts with paxillin and disrupts the actin cytoskeleton. Proc Natl Acad Sci USA 94:4412-4417

114. van Slegtenhorst M, Nellist M, Nagelkerken B, Cheadle J, Snell R, van den Ouweland A, Reuser A, Sampson J, Halley D, van der Sluijs P (1998) Interaction between hamartin and tuberin, the TSC1 and TSC2 gene products. Hum Mol Genet 7:1053-1057

115. Vogt M, Butz K, Dymalla S, Semzow J, Hoppe-Seyler F (2006) Inhibition of Bax activity is crucial for the antiapoptotic function of the human papillomavirus E6 oncoprotein. Oncogene 25:4009-4015

116. Wang GH, Bertin J, Wang Y, Martin DA, Wang J, Tomaselli KJ, Armstrong RC, Cohen JI (1997) Bovine herpesvirus 4 BORFE2 protein inhibits Fas- and tumor necrosis factor receptor 1-induced apoptosis and contains death effector domains shared with other gamma-2 herpesviruses. J Virol 71:8928-8932

117. Watanabe S, Kanda T, Yoshiike K (1989) Human papillomavirus type 16 transformation of primary human embryonic fibroblasts requires expression of open reading frames E6 and E7. J Virol 63:965-969

118. Wazer DE, Liu XL, Chu Q, Gao Q, Band V (1995) Immortalization of distinct human mammary epithelial cell types by human papilloma virus 16 E6 or E7. Proc Natl Acad Sci USA 92:3687-3691

119. Werness BA, Levine AJ, Howley PM (1990) Association of human papillomavirus types 16 and 18 E6 proteins with p53. Science 248:76-79

120. Zeng M, Kumar A, Meng G, Gao Q, Dimri G, Wazer D, Band H, Band V (2002) Human papilloma virus 16 E6 oncoprotein inhibits retinoic $\mathrm{X}$ receptor-mediated transactivation by targeting human ADA3 coactivator. J Biol Chem 277:45611-45618

121. Zimmermann H, Degenkolbe R, Bernard HU, O'Connor MJ (1999) The human papillomavirus type 16 E6 oncoprotein can down-regulate p53 activity by targeting the transcriptional coactivator CBP/p300. J Virol 73:6209-6219

122. zur Hausen H (2000) Papillomaviruses causing cancer: evasion from host-cell control in early events in carcinogenesis. J Natl Cancer Inst 92:690-698 